\title{
REVIEW OF THE WORLDWIDE SASE FEL DEVELOPMENT
}

\author{
Tsumoru Shintake
}

\section{RIKEN/SPring-8, Hyogo 679-5148 Japan.}

\section{Abstract}

Three major X-ray FELs are now under construction: LCLS[1] at SLAC Stanford, XFEL/SPring-8[2] in Japan and Euro-XFEL[3] at DESY Hamburg. They are all aiming at generating $1 \AA$ wavelength coherent X-ray radiation, which possess special feature: very high peak power and short pulse length. It will provide powerful tool to study non-linear dynamics of condensed matter, multi-photon ionization process of gases, and also it will realize the X-ray microscope of Angstrom resolving power, and instantaneous photography of femto-second time slicing capability. All of those XFELs rely on the same principle of SASE: Self-Amplified Spontaneous Emission, which does not require the cavity mirrors, thus wavelength is widely tuneable.

This paper reviews basic configuration of each machine and project status.

\section{INTRODUCTION}

In the past these ten years, extensive R\&D effort has been taken to realize X-ray FEL at various laboratories around the world, which was aiming at establishing key technologies required for SASE-FEL at Angstrom wavelength, and now technologies seem to be mature.

When X-ray beam is illuminated onto a condensed matter, it is absorbed due to the photo-ionization, and a small part plays Thomson scattering, which carries the structure information and cause Bragg's diffraction. As the X-ray wavelength becomes shorter, the photoionization cross-section reduces rapidly, and around $1 \AA$ they become comparable. This is the reason why $1 \AA$ wavelength is chosen in crystallography, and also in Xray FELs.

When a high energy electron beam runs through undulator: array of permanent magnet with alternating

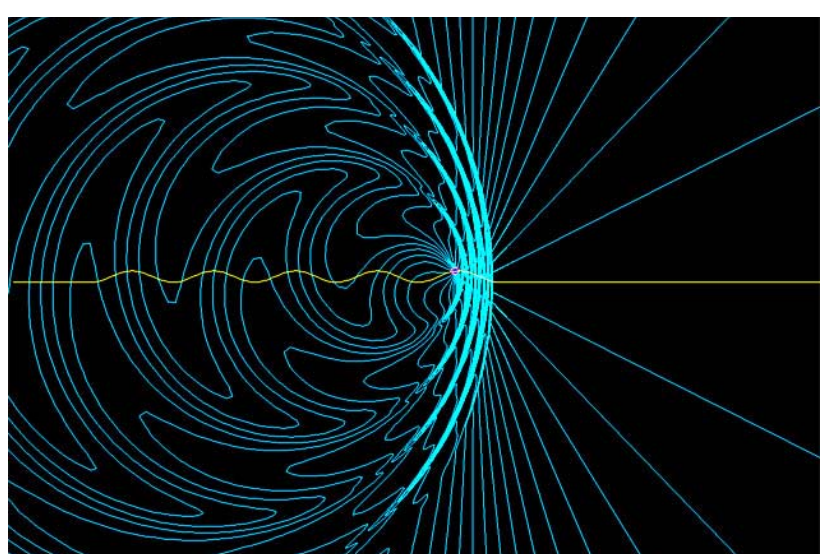

Fig. 1. X-ray FEL uses the undulator radiation. (a screen shot from Radiation2D) polarity, generates quasi-monochromatic radiation along the beam axis, which is called undulator radiation. This is graphically shown in Figure 1, which is given by using Radiation2D software[4]. The screen shot shows that the particle running along sinuous trajectory in the undulator, and radiates sinuous wave propagating forwardly on these electric field lines. Since the electron catches up the wave with close to speed of light, thus the wavelength is compressed due to Doppler's effect. It should be noted that the wave consists from series of spherical wavefronts, which approach to plane waves after propagating certain distance. Thus transverse coherency is always fairly good.

The wavelength is given by the following simple equation:

$$
\lambda=\frac{\lambda_{u}}{2 \gamma^{2}}\left(1+\frac{K^{2}}{2}\right),
$$

where $\lambda_{u}$ is the period of the magnet array, $\gamma$ is relativistic beam energy: $\gamma=1+E / m_{0} c^{2}, K$ represents intensity of undulator magnet $K=e B_{0} \lambda_{u} / 2 \pi m_{0} c$.

As a practical example, using $\lambda_{u}=3 \mathrm{~cm}, K=2$, to obtain $1 \AA$, we need $\gamma=20 \times 10^{3}$, which corresponds to the beam energy of $10 \mathrm{GeV}$. This is the reason why we need large scale high energy electron accelerator to obtain $1 \AA$ X-ray from undulators.

The above picture shows radiation from single electron, while in practical accelerators many electrons are flying together in a bunched form. Figure 2 shows the schematic drawing of radiation generated by group of electrons. In a storage ring, electrons runs random positions (Fig. 2 left), radiations from each electron will sometimes cancel or constructively build up, and as a result the total field

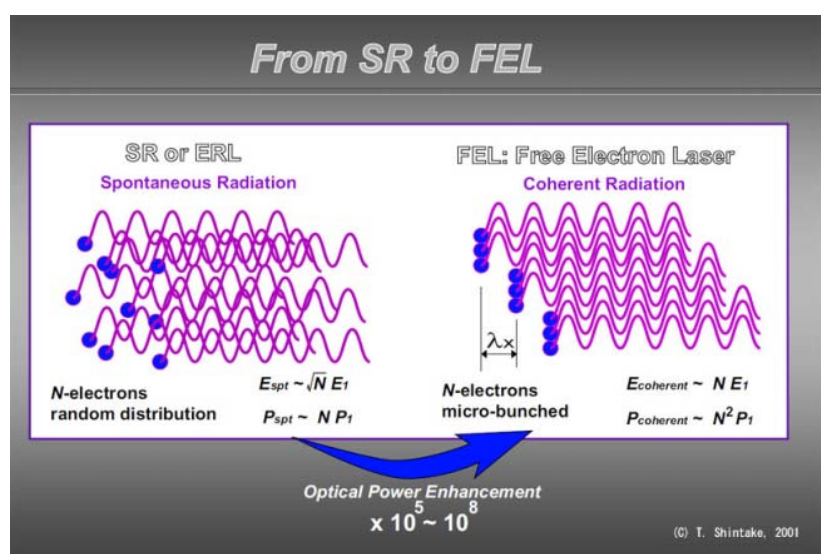

Fig. 2. Radiation patterns of spontaneous and coherent radiation modes.

\footnotetext{
\#shintake@spring8.or.jp
} 


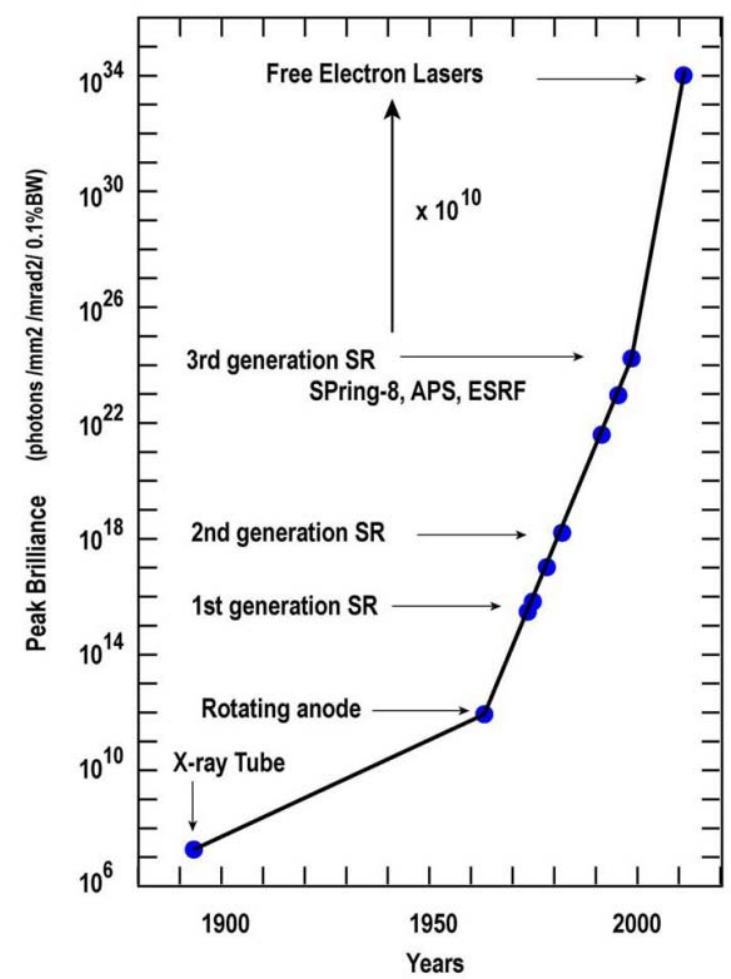

Fig. 3. Historical evolution of the peak brilliance in various $\mathrm{X}$-ray sources.

becomes statistical root-mean-square of each electron field. SR: synchrotron light source and ERL: energy recovery linac belong to this category.

If we align the longitudinal positions of these electrons regularly with the same spacing as the radiation wavelength, each radiation constructively builds up and the radiation field becomes very strong. The power gain after this effect ranges $10^{6}$ to $10^{8}$ according to the degree of density modulation. Including other factors which enhance the field intensity, i.e., smaller emittance and higher peak current, in total the peak brightness becomes $10^{10}$ times higher than highest brightness available in the $3^{\text {rd }}$ generation light synchrotron light sources. Figure 3 shows the historical evolution of peak brightness of the X-ray light sources. It started in 1895, when Röntgen found X-ray from cathode ray tube at university of Würzburg. Since then challenge to increase the X-ray intensity started. From 1960s, scientists stated to use synchrotron light from electron storage ring, thus the brightness increased very fast, from $1^{\text {st }}$ generation to $2^{\text {nd }}$ and finally $3^{\text {rd }}$ generation light sources, where the brightness has been reached to $10^{24}$ photons $/ \mathrm{mm}^{2} / \mathrm{mrad}^{2} / 0.1 \%$ bw. The recent top demand on high brightness has been the macro-molecular crystallography, which requires high brightness X-ray dose onto small protein sample, whose size is usually $100 \mu \mathrm{m}$ or even smaller, and it requires certain monochromatic spectrum and small divergence to meet sharp Bragg diffraction condition[5].

The longitudinal coherency of X-ray beam has not yet been playing important role in crystallography, since the $3 \mathrm{D}$ atomic array in a crystal acts as a narrow band monochrometer and it effectively lengthens the coherent length and creates Bragg diffraction in certain direction. The X-ray holography[6], which was extensively studied theoretically in 1970's to see atomic scale structure, it required coherent and high flux X-ray beam. However there was no such coherent source until now, thus it has not yet been realized in A resolution. Xray FEL will realize the dream of holography. One of the most challenging application is single molecular structural analysis based on diffraction microscopy technique[7].

The X-ray FEL will jump from today's $3^{\text {rd }}$ generation light source to $4^{\text {th }}$ generation light source by factor of 10 decades increase. However, it does not means that the Xray FEL will take over all the science around the today's light sources, instead it will provide complementary tool to explore new sciences which only attainable by means of its tremendous higher peak power and ultra-short pulse strobe light at femto-second range.

This paper does not discuss the scientific cases further in detail, but focus on the machine itself: basic design and different features between projects.

\section{SASE-FEL MACHINE}

Figure 4 shows schematic configuration of FELs. The upper drawing shows the conventional FEL with cavity resonator, mostly operates at visible or infrared wavelength. When the beam passes through the undulator, it generates monochromatic radiation, which is reflected back to upstream by the mirrors, and then it overlaps to the next electron beam. Interaction between electron and radiation in the undulator creates longitudinal periodic energy modulation, which is then converted to density modulation due to non-zero dispersion in the undulator magnet. Followed by radiation process and modulation feedback, the circulating power exponentially growths. Usually, the single path signal gain is fairly small, and it requires many turns of amplification process until it saturates.

\section{From Cavity Type FEL to SASE-FEL}

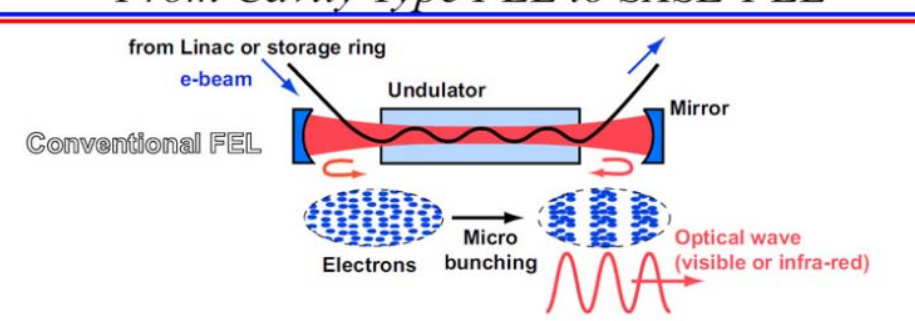

SASE口啊L

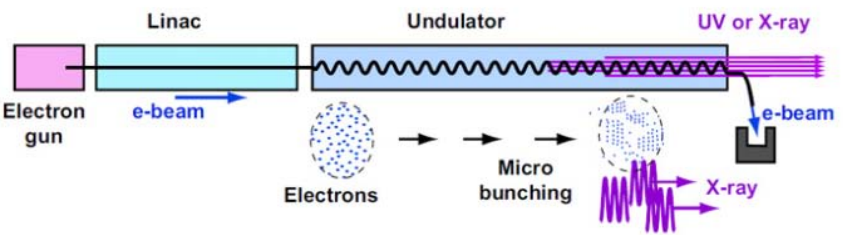

Fig. 4. Conventional FEL v.s. SASE-FEL. 
To make shorter the FEL wavelength, the reflection coefficient of resonator mirror becomes poor at $200 \mathrm{~nm}$ or shorter wavelength and also it is subject to be damaged due to high radiation power, as a result, this scheme becomes non feasible.

SASE-FEL solves this problem. As shown in Fig. 4 lower, if we feed a high density electron beam into long undulator, with small transverse emittance and low energy spread from linear accelerator, the optical FEL gain becomes high enough, and it can reach a saturation condition within a feasible undulator length. In this case, the signal starts from spontaneous radiation of upstream undulator, and it is amplified along the beam passage, thus it is named as Self-Amplified Spontaneous Emission: SASE. Since it does not require cavity resonator, there is no limit due to mirror performance, while it requires high quality and high density electron beam, and a long undulator line. Additionally it is required to control the electron beam in a straight trajectory with high precision. To reach saturation condition at $1 \AA \mathrm{X}$-ray, the typical beam parameter requirement is $3 \sim 6 \mathrm{kA}$ peak at $8 \sim 20$

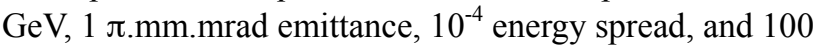
$\mathrm{m}$ long undulator, $10 \sim 30 \mu \mathrm{m}$ transverse trajectory error. They are highly technical challenging issues.

\section{TECHNOLOGY CHOICE}

In this section, technology choices on three major $\mathrm{X}$ ray FEL projects are discussed. We have to remind that actual technical decision is not always made by simple technical aspect, but fairly influenced by $R \& D$ history and regional factors, and also economical consideration is more important in realistic situation.

Table-1 shows the machine parameter list. It should be noted that definition on construction cost differs in each projects, and there is no straightforward comparison. Especially, LCLS project uses existing Two-mile Linac, while the other two projects need to construct linear accelerator and its tunnel from zero, thus the construction cost must be much differ.

Three projects use different type of undulator technologies, which reflect back to the magnet period and required beam energy. SCSS XFEL/Spring- 8 use short period in-vacuum type undulator, thus the electron beam energy is lower than the other two.

\section{Low Emittance Electron Source}

LCLS uses rf photo-cathode gun driven by S-band pulse power, see Fig. 5 (a). Focusing solenoid is closely mounted to the cavity for compensating phase space ellipse rotation. Bare cupper is used as the photo-cathode. Recently, LCLS team successfully injected beam into the linac, and verified the beam quality. Thanks to deflection cavity installed in the injector, the slice beam parameter is carefully measured and verified [8].

Euro-XFEL uses rf gun of normal conducting rf cavity at L-band $1.3 \mathrm{GHz}$. CeTe is used to provide high quantum efficiency to produce multi-bunch beam. The system is operating nicely and providing multi-bunch in daily operation for FLASH experiment[9]. One problem is large dark current from the cathode, which propagates down the linac and activates component around chicane. $\mathrm{R} \& \mathrm{D}$ for collimator and rf kicker are undertaken[10].

Alternative technology has been development at SCSS SPring-8, as shown Fig. 5 (c) where the single crystal thermionic cathode is used to generate low emittance DC beam at relatively low beam current, then compressed by velocity bunching in the injector with $\mathrm{rf}$ energy modulation[11]. By careful control of beam bunching and transverse beam matching, the first lasing was achieved at $49 \mathrm{~nm}$ in 2006 June. It has been demonstrated that 400 times compression without significant emittance deterioration[12]. For the XFEL/Spring-8 project, another factor of ten compression is required, which will be straightforwardly obtained by introducing second magnetic chicane bunch compression systems.

\section{Main Accelerator}

Task of the main accelerator system is to accelerate beam up to high energy around $8 \sim 20 \mathrm{GeV}$. Euro-XFEL uses the super conducting technology at $1.3 \mathrm{GHz} \mathrm{L}$-band, which is now frequently chosen in many other proposals for SASE FELs at soft X-ray wavelength. Major benefit of the super conducting technology is its long pulse acceleration or CW beam acceleration capability, while its has a draw back of higher construction cost (because of

Table-1 Machine parameter of X-ray FELs.

\begin{tabular}{|l|c|c|c|}
\hline Projects & Euro-XFEL & LCLS (SLAC) & XFEL/SPring8 (SCSS) \\
\hline Wavelength & $6-0.085 \mathrm{~nm}$ & $1.5-0.15 \mathrm{~nm}$ & $6-0.08 \mathrm{~nm}$ \\
\hline Beam Energy & $10-20 \mathrm{GeV}$ & $14.3 \mathrm{GeV}$ & $2-8 \mathrm{GeV}$ \\
\hline Main Accelerator & Super Conducting & S-band Normal Conducting & C-band Normal Conducting \\
\hline Accelerator Length & $2.1 \mathrm{~km}$ & $1 \mathrm{~km}$ & $400 \mathrm{~m}$ \\
Gradient x Active Length & $23.5 \mathrm{MV} / \mathrm{m} \mathrm{x} 900 \mathrm{~m}$ & $19 \mathrm{MV} / \mathrm{m} \mathrm{x} 800 \mathrm{~m}$ & $35 \mathrm{MV} / \mathrm{m} \mathrm{x} \mathrm{230} \mathrm{m}$ \\
\hline Undulator Period & $26 \mathrm{~mm}$ & $30 \mathrm{~mm}$ & $18 \mathrm{~mm}$ \\
Total undulator Length & $133 \mathrm{~m}$ & $113 \mathrm{~m}$ & $90 \mathrm{~m}$ \\
\hline Total Length & $3.4 \mathrm{~km}$ & $1.6 \mathrm{~km}$ & $700 \mathrm{~m}$ \\
Undulator Lines (X-ray) & $3(5)$ & $1(5)$ & $1(3), \mathrm{max} 5$ \\
\hline Construction Cost & $850 \mathrm{M}-$ Euro & $380 \mathrm{M} \$$ & $300 \mathrm{M} \$$ \\
\hline
\end{tabular}




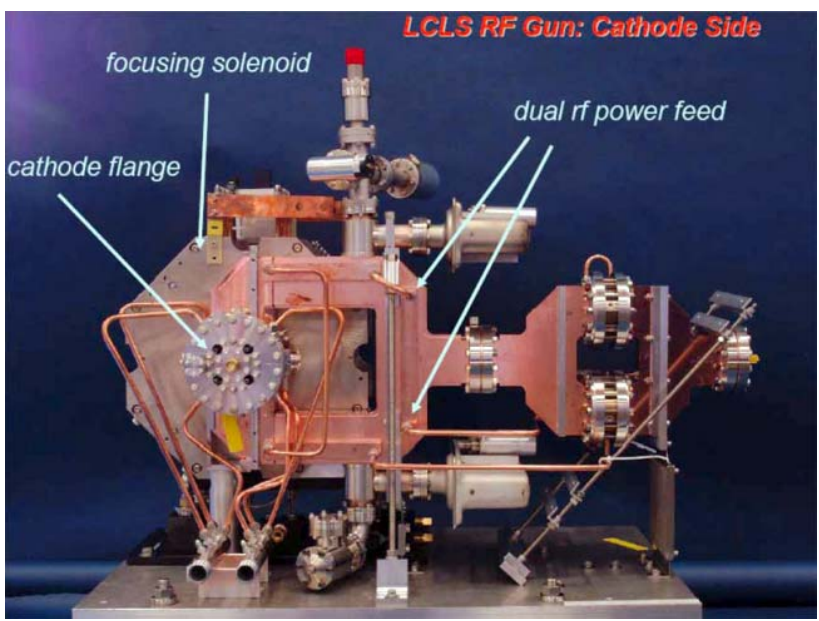

(a) LCLS rf gun.

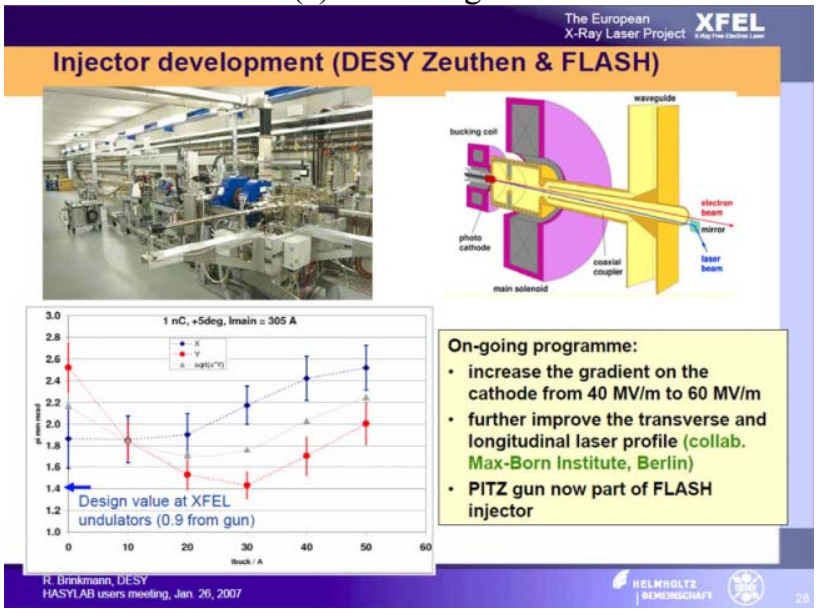

(b) FLASH \& Euro-XFEL rf gun.

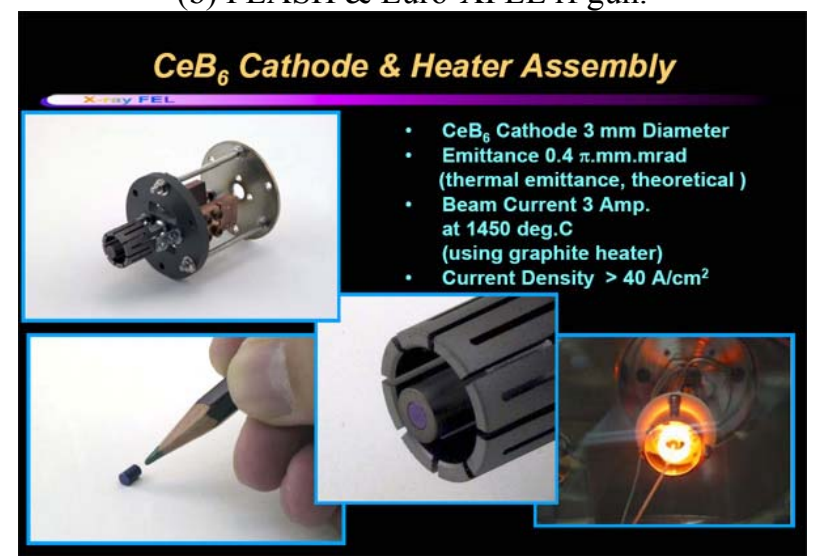

(c) $\mathrm{CeB}_{6}$ single crystal thermionic cathode.

Fig. 5. Three different types of electron sources.

complex structure and lower gradient in $\mathrm{CW}$ operation thus longer accelerator.

The normal conducting accelerator at S-band frequency is still commonly used because of its well established technology and lower construction cot (simple structure and higher gradient thus shorter accelerator). Most challenging accelerator in normal conducting technology is C-band (twice of S-band frequency) accelerator system, which was originally developed for main accelerator technology in $\mathrm{e}^{+} \mathrm{e}^{-}$linear collider project[Shintake]. The first C-band system has been employed in SCSS test accelerator and running at $35 \mathrm{MV} / \mathrm{m}$ accelerating gradient in daily operation[13].

Technically speaking, if we operate X-ray FEL as single bunch machine, the normal conducting technology will be the best choice. In case of $\mathrm{CW}$ beam, if some application requires higher average flux, the super conducting technology will be necessary. However, we have to remind that as we discussed in previous section, special feature of X-ray FEL is its ultra-high peak brilliance beam, not the average flux, thus single shot operation is default mode in X-ray FELs. Since there are conventional synchrotron light sources, which generate high average flux in daily operation, the average brightness is not an important feature requested in X-ray FEL at the moment. And also, using high peak power, too high diffraction X-ray from a sample will saturate CCD detector with single short pulse. Also, to read out the image needs certain access time, especially for a large format CCD it becomes fairly slow: $1 \mathrm{sec}$ or longer, which limits the machine operation cycle.

For a long term perspective, if we conduct dedicated $\mathrm{R} \& \mathrm{D}$ for detector technology, it will become possible to fully use $\mathrm{kHz}$ bunch train from super conducting technology.

\section{Undulator}

Most of all projects employ permanent magnet undulator. There is attractive alternative technology exist: the super conducting magnet or microwave undulator[14]. They can provide short-period and higher field, however up to now, there is no example practically used in X-ray FELs.

There are two types of permanent magnet undulator, i.e., out vacuum and in vacuum design. The out vacuum design uses two magnet array at outside the vacuum pipe. Benefit of this design is that magnet assembly design is much simpler than in vacuum design, and field measurement and tuning of magnet array can be made independent from the vacuum pipe. In stead its draw back is that the magnetic gap has to be large enough to house the vacuum pipe within the gap, as a result, the undulator period becomes longer than in vacuum design. Since FEL gain is proportional to the K-parameter of magnet, longer period is desirable, but it reflects back to higher beam energy and longer accelerator.

SCSS XFEL/SPring-8 uses in vacuum design[15]. Benefit of this design is that the permanent magnet array sits inside vacuum tank, there is no vacuum envelop between the gap, thus we may bring the magnet pole closer to the beam, as a result the maximum available field become higher than the out vacuum design. Since vacuum acts as a good thermal insulator, the magnet temperature is well isolated from environmental temperature change, just like a vacuum bottle. Neodymium iron boron $(\mathrm{NdFeB})$ magnet is frequently used as undulator magnet because of its high remanent field and coercive force, and lower cost. However the remanent field has relatively high temperature coefficient $(-0.1 \% / \mathrm{deg})$, therefore it is important to control the 
magnet temperature within 0.1 degree C. A draw back of the in vacuum design is restricted access to the magnet array, thus lower maintainability.

\section{DISUSSIONS ON}

\section{TECHNICAL ISSUES LEFT BEHINED Dark Current}

In the rf-photo cathode gun, the high density electron beam is directly generated by short pulse laser illuminating on cathode material. In order to prevent the beam from emittance break due to the space-charge force, we apply high accelerating gradient on the cathode, which however causes dark-current emission from the cathode surface. The dark current fills all rf buckets, thus average current or total charge in one pulse becomes large enough, and sometimes larger than the photo-emitted main beam. Such dark-current from the cathode occupies the same transverse phase-space of photo-emitted beam, and has large energy spread. It propagates down through the accelerator and makes high radiation dose at the chicane area, or cause radiation damage on the permanent magnets in the undulator. Therefore, careful design of beam collimators is required[10].

In the thermionic gun case, the accelerating gradient is low, thus there is no dark current emission from the cathode surface, in stead, the main beam itself creates hallow beam during the velocity compression process at the injector. Therefore variable gap collimators have been installed injectors in the SCSS test accelerator, which works properly and we do not see high radiation does downstream.

Because the dark current from the accelerating structure has different phase volume from the main beam in $6 \mathrm{D}$ phase space, this type of dark current can be cut out easily. It is important to prepare a dog-leg or chicane to provide energy filter to clean up the dark current.

\section{Machine Stability}

SASE has intrinsic intensity fluctuation in pulse-topulse mode. When FEL power reaches saturation, the fluctuation level becomes lower, typically as low as $30 \%$. Even with this fluctuation, we may perform most scientific applications by monitoring the power level or averaging the signal over many shots.

The machine stability requirement is to maintain the saturation condition. In practical machine, this condition is easily broken by the various parameter changes:

Generally speaking, the upstream components have more strong effect on beam parameter change. In our SCSS case, the requirement on rf voltage and phase is typically $10^{-4}$ and 0.1 degree. In today's accelerator technology, this is feasible level by introducing feed back loops. However, it can not rescuer the pulse-to-pulse fluctuation, thus $R \& D$ is required eliminate jitters.

\section{CONCLUSION}

Technologically, the author does not see obvious obstacle to realizing FEL at $1 \AA$ wavelength. The first Xray FEL will soon be in operation from 2009 at LCLS. A few years later, the other two projects will start to provide laser X-ray beam to the user community.

However, discussions will be necessary to evaluate the construction cost of X-ray FEL machine. As listed in Table-1, they are rather expensive machines comparing with storage ring type light sources. The real value depends on the scientific products. We do not know yet how much the XFEL will bring new science. Therefore we have to wait ten years or more before to know fair justification.

\section{REFERENCES}

[1] LCLS web-site: www-ssrl.slac.stanford.edu/lcls/

[2] XFEL/Spring8 and SCSS Web-site: wwwxfel.spring8.or.jp.

[3] EuroXFEL: Web-site: www.xfel.net/en/index.html

[4] T. Shintake, "New Mathematical Method for Radiation Field of Moving Charge", EPAC02, Paris, France, 2002.

[5] Colin Nave, "Matching X-ray source, optics and detectors to protein crystallography requirements", Acta Cryst. D55, 1663-1668, 1999

[6] R. VanLigten and H. Osterberg, "Holographic Microscopy", Nature 211, 282-283, 1966,

J. C. Solem and G. F. Chapline, "X-ray biomicropholography", Opt. Eng. 23, 193-203 (1984)

[7] D. Sayre, "Possibility of Phase Retrieval by Oversampling X-ray Diffraction Pattern", Acta Cryst. 5, 843 (1952), N. H. Chapman et. al., Nature Physics 2, 839 (2006)

[8] Paul Emma et. al., "Initial Commissioning Experience with the LCLS Injector", at this conference.

[9] L. Monaco, et. al, "High QE Photocathodes Performance During Operation at FLASH/PITZ Photoinjectors", at this conference.

[10] L. Frohlich, "Dark Current Transport in the FLASH Linac", at this conference..

[11] K. Togawa, et. al., "CeB6 electron gun for lowemittance injector", Phys. Rev. ST Accel. Beams 10, 020703 (2007), T. Shintake, et. al, "SPring-8 Compact SASE Source", Proceedings of SPIE, Optics for Fourth-Generation X-Ray Sources, edited by R. O. Tatchyn, A. K. Freund, and T. Matsushita (SPIE, Bellingham, WA, 2001), Vol. 4500, pp. 12-23.

[12] H. Tanaka et al., "Low Emittance Injector at SCSS", FEL2006 conference, Berlin, August, 2006.

[13] T. Inagaki, et.al, "C-band Linac in SCSS Prototype Accelerator of the Japnanese X-FEL Project", at this conference.

[14] T. Shintake et al., "Microwave Undulator", Japanese Journal of Applied Physics Letters Vol.21, No.10, pp. L601-L603.

[15] T. Tanaka et.al., "In-Vacuum Undulators", FEL2005, Stanford CA, USA, Aug. 21-16, 2005. 